\title{
Swirl-nozzle interaction experiment: quasi-steady model-based analysis
}

\author{
Lionel Hirschberg $^{1}$ (D) $\cdot$ Friedrich Bake $^{1} \cdot$ Karsten Knobloch $^{1} \cdot$ Angelo Rudolphi $^{1} \cdot$ Sebastian Kruck ${ }^{1}$. Oliver Klose ${ }^{1}$. \\ Steven J. Hulshoff ${ }^{2}$
}

Received: 30 March 2021 / Revised: 17 July 2021 / Accepted: 19 July 2021 / Published online: 27 July 2021

(c) The Author(s) 2021

\begin{abstract}
Measurements of sound due to swirl-nozzle interaction are presented. In the experiment a swirl structure was generated by means of unsteady tangential injection into a steady swirl-free flow upstream from a choked convergent-divergent nozzle. Ingestion of swirl by the choked nozzle caused a mass-flow rate change, which resulted in a downstream-measured acoustic response. The downstream acoustic pressure was found to remain negative as long as the swirl is maintained and reflections from the open downstream pipe termination do not interfere. The amplitude of this initial acoustic response was found to be proportional to the square of the tangential mass-flow rate used to generate swirl. When the tangential injection valve was closed, the mass-flow rate through the nozzle increased, resulting in an increase of the downstream acoustic pressure. This increase in signal was compared to the prediction of an empirical quasi-steady model, constructed from steady-state flow measurements. As the opening time of the valve was varied, the signal due to swirl evacuation showed an initial overshoot with respect to quasi-steady behavior, after which it gradually decayed to quasi-steady behavior for tangential injection times long compared to the convection time in the pipe upstream of the nozzle. This demonstrates that the acoustic signal can be used to obtain quantitative information concerning the time dependence of the swirl in the system. This could be useful for understanding the dynamics of flow in engines with swirl-stabilized combustion.
\end{abstract}

This work was carried out while Lionel Hirschberg was the beneficiary of a Deutsches Zentrum für Luft- und Raumfahrt (DLR) - Deutscher Akademischer Austauschdienst (DAAD) postdoctoral fellowship (No. 57424730).

Extended author information available on the last page of the article 


\section{Graphic abstract}

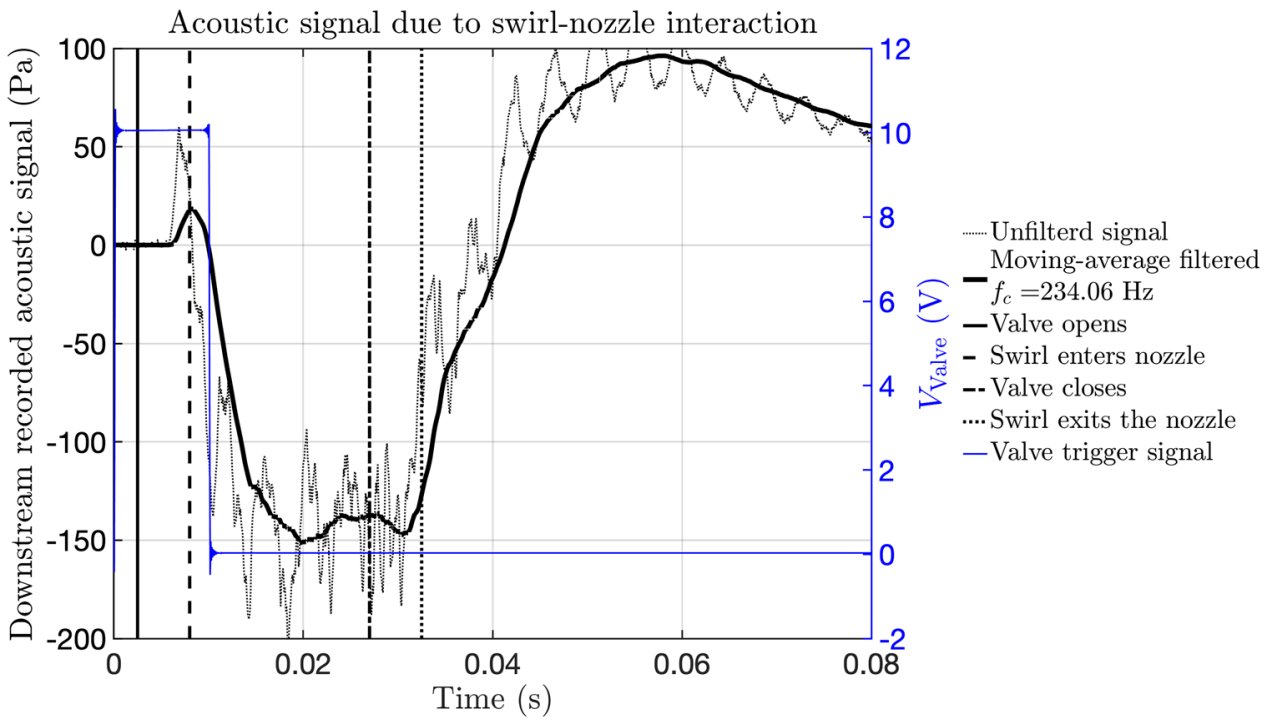

\section{Introduction}

Engineering systems employing turbulent combustion usually have high levels of noise production, due to both direct and indirect combustion-noise sources. Direct sources, due to unsteady gas expansion in flames, have been widely studied (Strahle 1971; Dowling and Mahmoudi 2015; Morgans and Duran 2016; Ihme 2017). Indirect sources include entropy noise, caused by entropy patches (localized nonuniformity in the fluid's thermodynamic state), and vorticity noise, caused by vortices. Entropy patches and vortices produce sound waves as they exit the area of combustion through a nozzle or turbine. Some of these are radiated into the environment, and some are reflected back into the combustion chamber. The latter can induce the production of new entropy patches and/or vortices. Under certain circumstances, this results in a feedback loop that promotes combustion instability. Indirect-combustion noise-driven thermoacoustic combustion-chamber instability is a problem in aeroengines, electrical-power generation turbines and solid rocket motors (Dowling and Mahmoudi 2015; Morgans and Duran 2016; Dotson et al 1997; Hulshoff et al 2001; Anthoine et al 2002; Hirschberg et al 2018a, b, 2019; Hirschberg and Hulshoff 2020). Entropy noise has been widely studied, as evidenced by the high number of citations of two seminal articles by Marble and Candel (1977) and Ffowcs Williams and Howe (1975). Vorticity noise has received far less attention.

In the case of vorticity noise, one should distinguish between sound produced by the interaction of a vortex oriented normal to the main flow with a nozzle, and that produced by the interaction of a vortex oriented parallel (swirl) to the main flow with a nozzle.

Sound production due to the interaction of a choked nozzle with vorticity oriented normal to the main flow, is known to be an essentially unsteady mechanism. This occurs, for example, when azimuthal ring vortices are shed at thermal inhibitors in Solid Rocket Motors (Hulshoff et al 2001; Anthoine et al 2002; Hirschberg et al 2018a, b, 2019; Hirschberg and Hulshoff 2020; Hirschberg et al 2021b).

Most combustors used in gas turbines and aeroengines are swirl-stabilized, i.e., in these a significant vorticity component is created parallel to the main flow. Kings and Bake (2010) performed cold-gas experiments to investigate parallel-component vorticity noise experimentally in isolation. This was done by means of pulsated tangential air injections into a steady flow upstream from a choked nozzle.

Hirschberg et al (2021b) argued that in this experiment, sound production is due to a reduction of the mass-flow rate through the nozzle as the upstream-generated swirling flow structure is ingested. The analysis of Hirschberg et al (2021b) employed a quasi-steady quasi-cylindrical analytical model, which indicated that the upstream swirlintensity change, $\Delta S$, due to unsteady tangential injection was the driving parameter for sound production. The definition of swirl, $S$, used by Hirschberg et al (2021b) was taken from Gany et al (2005), and is the most commonly found in the literature. This is qualitatively understood to be the ratio of angular moment flux and axial momentum flux. Analytical modeling by Gany et al (2005) and van 
Holten et al (2009) shows a quadratic dependence of the reduction of axial mass-flow rate through a choked-nozzle throat on the swirl $S$. Hirschberg et al (2021b) proposed an acoustic model of the experiments. The acoustic model yielded the following scaling rule: $\Delta p_{2}^{\prime} \propto \Delta S^{2}$, where $\Delta p_{2}^{\prime}$ is the amplitude of the acoustic signal due to swirl-nozzle interaction measured downstream of the choked nozzle. In a discussion of various definitions of swirl, $S$, Carpenter (1976) argues that this classical definition of $S$ is not necessarily related to the mass-flow rate reduction upon ingestion of a swirl structure.

Using a different definition of swirl, Dutton (1989) empirically and numerically found a quadratic dependence of the relative mass-flow rate on the magnitude of swirl for steady flow through a choked nozzle at constant reservoir conditions. In Dutton (1989), the swirl, $S_{\text {Dutton }}$ is defined as the ratio $S_{D}$ of the cross-sectional average of the azimuthal velocity at the throat and the critical speed of sound $c^{*}$. Carpenter (1976) concluded that the mass-flow rate reduction through a choked nozzle scales as the crosssectional average of the ratio of the square of the azimuthal velocity at the throat $\left\langle u_{\theta, \mathrm{th}}^{2}\right\rangle$ divided by $\left(c^{*}\right)^{2}$. In this text, a quasi-steady quasi-cylindrical analytical model assuming a uniform azimuthal velocity $u_{\theta, \text { th }}$ is considered. This corresponds to the constant angle model of Dutton (1989). Due to axial elongation of the swirl when it passes through the nozzle, the azimuthal velocity, $u_{\theta}$, will increase due to the conservation of angular momentum. This reduces the enthalpy available for axial acceleration, and due to the increased Mach number, lowers the density at the throat, reducing the mass-flow rate for given reservoir conditions. This explanation favors the choice of Carpenter (1976). For the definition of the swirl number, however, to facilitate comparison with the data provided by Dutton (1989), Dutton's definition of swirl number will be used. Furthermore, when considering a rocket engine, Carpenter (1976) is less certain of the best definition of swirl number, while
Dutton (1989) claims that his definition provides a better collapse of data.

Recently, Hirschberg et al (2021a) performed swirl-nozzle interaction experiments with an improved version of the setup used by Kings and Bake (2010). A sketch of the setup is shown in Fig. 1. These improvements included the use of an unsteady tangential-injection reservoir of precisely defined volume $V_{\theta}=2.8 \times 10^{-3} \mathrm{~m}^{3}$. A steady-flow calibration was performed to relate the unsteady tangentialinjection reservoir pressure $p_{\theta}$ to the unsteady tangential mass-flow rate $\dot{m}_{\theta}$. Upstream from the convergent-divergent nozzle a microphone was added (Fig. 1). This made it possible to measure the evolution of the reservoir pressure $p_{1}$ due to unsteady tangential injection. The outlet section downstream of the microphone section was extended by three meters, to facilitate the quantitative measurement of the acoustic signal due to swirl-nozzle interaction. However, the time span available for reflection-free measurement was only ca. $30 \mathrm{~ms}$, the time it took for an acoustic signal to travel from the downstream microphone to the open-pipe termination and back.

In the present study, acoustic signals due to both ingestion and evacuation of the axially-oriented vortex are considered. In particular, the influence of the duration of the tangential-injection time on these signals is investigated. The downstream section was also further extended, to obtain in total a 24-m-long section of flexible tube downstream of the downstream microphone (Fig. 1). This was done to ensure a longer, ca. $140 \mathrm{~ms}$, reflection-free measurement of the swirl-nozzle interaction signal. A detailed description of the experimental setup used to obtain the results analyzed here is provided in Sect. 3.

Hirschberg et al (2021a) asserted that in their experiment one observes quasi-steady behavior. They argued that even the shortest swirl structures considered were long (in the axial direction) compared to the nozzle length. This suggests steady-flow measurements of swirl-nozzle interaction can be used to build an empirical quasi-steady model for the prediction of the downstream acoustic response due to unsteady
Fig. 1 Sketch of swirl-nozzle interaction setup

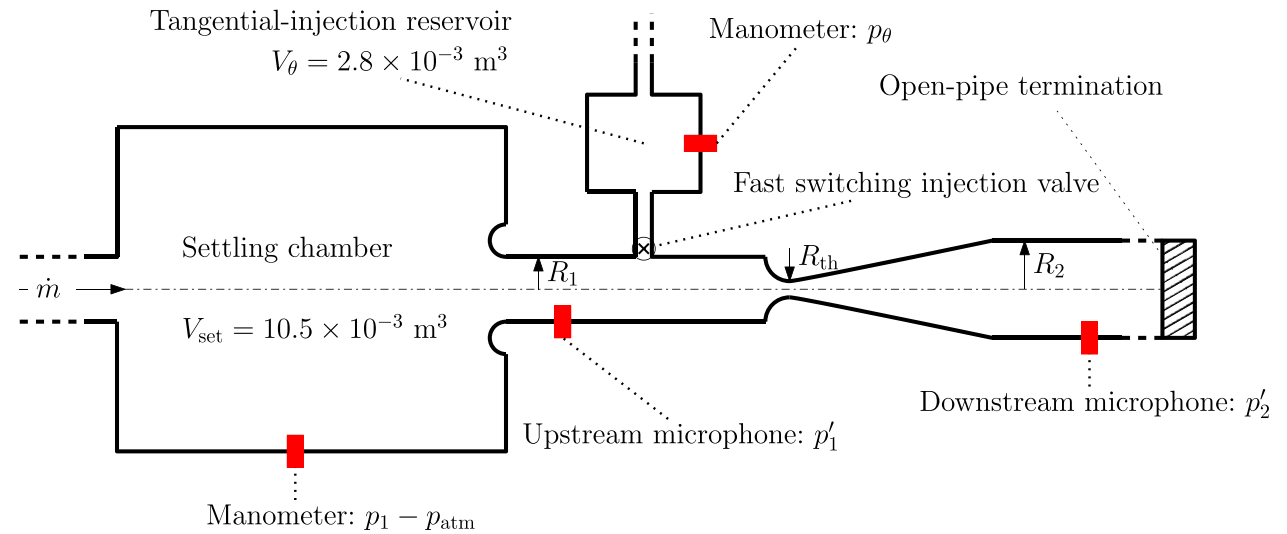


swirl-nozzle interaction. This modeling strategy is applied in this text. To this end, it will be shown that steady-state flow measurements allow the establishment of an empirical relationship between the tangentially-injected mass-flow rate $\dot{m}_{\theta}$ and the total axial mass-flow rate through the nozzle, $\dot{m}_{\text {tot }}$ as a function of the upstream reservoir pressure $p_{1}$.

In Sect. 2, a review of literature concerning the definition of swirl and the effect of swirl on a steady flow through a choked nozzle is provided (Sect. 2.1). This is followed by a proposed empirical quasi-steady model for the sound emission downstream from the nozzle (Sect. 2.2).

The experimental setups and measurement procedures used for the acoustic measurements (tangential and radial injection) and steady-state flow measurements are described in Sects. 3.1, and 3.2, respectively. The unsteady mass-flow rate $\dot{m}_{\theta}$ calibration is described in "Appendix 2".

The results of the steady-state flow measurements are presented in Sect. 4.1, while the unsteady acoustic measurement results are discussed in Sect. 4.2. A typical acoustic measurement result is discussed in Sect. 4.2.1. Both upstream and downstream effects of the opening and closing of the tangential-injection valve are shown and analyzed in Sect. 4.2.1. By means of the typical acoustic measurement, complemented by radial mass injection experiments ("Appendix 3"), direct sound production (not involving an flow-nozzle interaction) is shown to be insignificant. A detailed analysis of direct sound in the experiments is provided in "Appendix 3".

The downstream-measured amplitude $\Delta p_{2}^{\prime}$, due to the swirl ingestion by the nozzle and its subsequent evacuation are compared to the prediction of the empirical quasi-steady theory in Sect. 4.2.3. The effect of the tangential-injection time $\tau_{\theta}$ is analyzed. The scaling law proposed by Hirschberg et al (2021a), which assumes that swirl-nozzle interaction sound production is proportional to the square of the tangentially injected mass-flow rate, is revisited and its limitations are discussed.

\section{Theory}

\subsection{Steady swirl-nozzle interaction}

The commonly-used definition of swirl $S$, in a pipe flow is the ratio of angular momentum flux to axial momentum flux. Assuming an axisymmetrical flow in a pipe of radius $R$, one has (Hirschberg et al 2021b; Gany et al 2005)

$S \equiv \frac{\int_{0}^{R} u_{\theta} u_{x} r^{2} \mathrm{~d} r}{R \int_{0}^{R} u_{x}^{2} r \mathrm{~d} r}$

where $u_{\theta}$ is the azimuthal velocity, $u_{x}$ is the axial velocity, and $r$ is the distance to the pipe axis. This definition was used by Hirschberg et al (2021b), but will not be used in this text for the reasons explained in the introduction.

The relative reduction of the axial mass-flow rate $\Delta \dot{m}_{\mathrm{st}} / \dot{m}^{*}=\left(\dot{m}_{\mathrm{st}}-\dot{m}^{*}\right) / \dot{m}^{*}$, of a choked-nozzle flow due to the presence of the swirl in the nozzle is for an isentropic flow with uniform azimuthal velocity $u_{\theta, \text { th }}$ at the throat:

$\frac{\Delta \dot{m}_{\mathrm{st}}}{\dot{m}^{*}}=\left(1-\frac{\gamma-1}{\gamma+1}\left(\frac{u_{\theta, \mathrm{th}}}{c^{*}}\right)^{2}\right)^{\frac{\gamma+1}{2(\gamma-1)}}-1$

with

$\dot{m}^{*}=\rho^{*} c^{*} A_{\mathrm{th}}$

where $c^{*}$ is the critical speed of sound, $\rho^{*}$ the critical density, $A_{\mathrm{th}}=\pi R_{\mathrm{th}}^{2}$ is the choked nozzle throat cross-sectional area, and $\gamma=1.4$ is the heat capacity ratio of air (assumed to be an ideal gas with constant specific heat). This analytical result is obtained by assuming a quasi-cylindrical isentropic flow with uniform $u_{\theta}$ over a cross section, but varying along the axial coordinate of the duct (details are provided in "Appendix 1"). The expression $\Delta \dot{m}_{\mathrm{st}} / \dot{m}=-S_{\text {Dutton }}^{2} / 2$ used by Hirschberg et al (2021a) is the first-order Taylor expansion of Eq. (2) for small values of $S_{\text {Dutton }}^{2}$.

Clearly, a uniform azimuthal velocity is not physically realistic. However, the results obtained by Gany et al (2005) or Dutton (1989) for more realistic azimuthal velocity profiles show a deviation of at most $20 \%$ from this assumption. Dutton (1989) proposed the use of the cross-sectional area average azimuthal velocity at the throat

$\left\langle u_{\theta, \mathrm{th}}\right\rangle \equiv \frac{2}{R_{\mathrm{th}}^{2}} \int_{0}^{R_{\mathrm{th}}} u_{\theta, \mathrm{th}} r \mathrm{~d} r$

divided by the critical speed of sound $c^{*}$ as an alternative definition of swirl

$S_{\text {Dutton }} \equiv \frac{\left\langle u_{\theta, \mathrm{th}}\right\rangle}{c^{*}}$.

N.b., for a uniform azimuthal velocity $\left\langle u_{\theta, \mathrm{th}}\right\rangle=u_{\theta, \mathrm{th}}$, $S_{\text {Dutton }}=u_{\theta} / c^{*}$. Using Eq. (5), Dutton (1989) obtained a fit of numerical and experimental data, including that of a free vortex. However, one notes that the theory predicts only a quadratic dependence of $\Delta \dot{m}_{\text {st }} / \dot{m}$ on $S_{\text {Dutton }}$ (Eq. (2)). Thus, a simplification which omits the linear term of the data fit reported by Dutton (1989) is proposed :

$\frac{\Delta \dot{m}_{\mathrm{st}}}{\dot{m}^{*}}=-0.45\left(\frac{\left\langle u_{\theta, \mathrm{th}}\right\rangle}{c^{*}}\right)^{2}=-0.45 S_{\text {Dutton }}^{2}$

As can be seen in Fig. 2, Eq. (2) agrees with the proposed correlation (Eq. (6)) within ca. $6 \%$ in $\Delta \dot{m}_{\mathrm{st}}$ for $\left\langle u_{\theta, \mathrm{th}}\right\rangle / c^{*}=0.5$. One observes that this is actually within 
Fig. 2 Data reported by Dutton (1989) fitted using Eq. (6), and compared to the theoretical quasi-steady model Eq. (2)

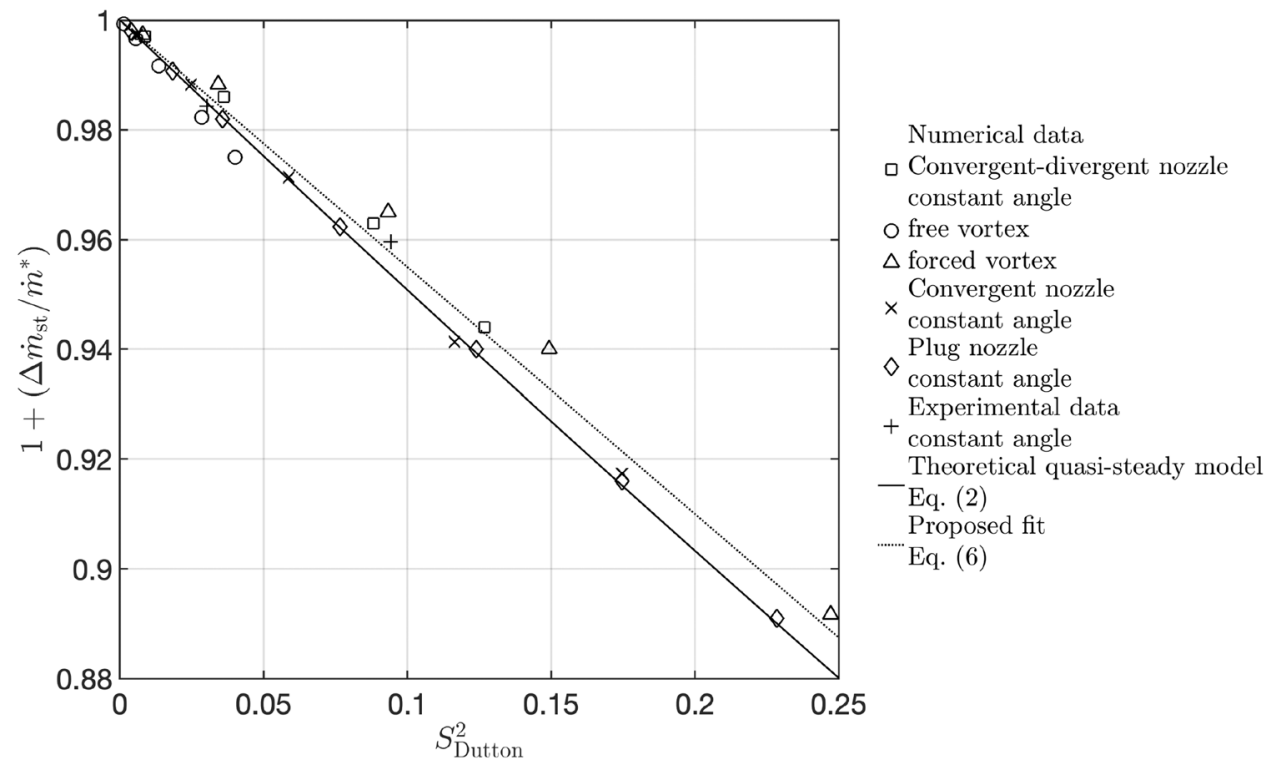

the deviations observed between the various data sets of Dutton (1989).

\subsection{Acoustics model}

Swirl-nozzle interaction sound production occurs due to an abrupt mass-flow rate change due to a change in swirl in the choked nozzle throat (Hirschberg et al 2021b, a). An abrupt mass-flow rate decrease causes an expansion pressure signal to be emitted downstream from the choked nozzle. It will be shown that the ingestion of swirl causes such an abrupt mass-flow rage change. A compression pressure signal is emitted downstream when where the mass-flow rate is increased abruptly. This will be shown to occur when upstream-generated swirl is evacuated by the nozzle. In this section an acoustic model is proposed based on these observations.

The acoustic amplitude $\Delta p_{2}^{\prime}$ generated downstream of the choked nozzle for anechoic downstream conditions is related to the variation in downstream flow velocity $u_{2}^{\prime}$, as follows (Hirschberg et al 2021a):

$\Delta p_{2}^{\prime}=\rho_{2} c_{2} u_{2}^{\prime} \simeq \frac{c_{2} \Delta \dot{m}}{A_{2}}$

where $A_{2}=\pi R_{2}^{2}$ is the cross-sectional area of the downstream pipe, and $\rho_{2}$ and $c_{2}$ are the density and speed of sound downstream from the nozzle.

The variation $\Delta \dot{m}$ due to a quasi-steady variation in $\dot{m}_{\theta}$ can be estimated from a fit of the steady-flow data $\dot{m}_{\text {fit }}\left(p_{1},\left(\dot{m}_{\theta} / \dot{m}\right)^{2}\right)$, as follows:

$\Delta \dot{m} \simeq \dot{m}_{\mathrm{fit}}\left(p_{1},\left(\dot{m}_{\theta} / \dot{m}\right)^{2}\right)-\dot{m}_{\mathrm{fit}}\left(p_{1}, 0\right)$ where $\dot{m}_{\text {fit }}\left(p_{1}, 0\right)$ is the mass-flow rate in the absence of tangential injection and $\dot{m}_{\mathrm{fit}}\left(p_{1},\left(\dot{m}_{\theta} / \dot{m}\right)^{2}\right)$ is the mass-flow rate with tangential injection at the same pressure $p_{1}$. A simplification used in the analysis of the experiments is that for the short injection times considered, here the reservoir pressure $p_{1}$ will, because of the large reservoir volume $V_{\text {set }}$, not significantly change. The variation in $\dot{m}$ is neglected when estimating $\dot{m}_{\theta} / \dot{m}$. Details of the experimental setup used to obtain the steady-state data for $\dot{m}_{\text {fit }}\left(p_{1},\left(\dot{m}_{\theta} / \dot{m}\right)^{2}\right)$ are described in Sect. 3.2. The steady-state flow measurement results are reported in Sect. 4.1. The resulting empirical quasi-steady model (QSM)

$\Delta p_{2, \mathrm{QSM}}^{\prime} \simeq \frac{c_{2}}{A_{2}}\left(\dot{m}_{\mathrm{fit}}\left(p_{1},\left(\dot{m}_{\theta} / \dot{m}\right)^{2}\right)-\dot{m}_{\mathrm{fit}}\left(p_{1}, 0\right)\right)$

is compared to and used to scale acoustic-measurement results in Sect. 4.2.3. Steps used to derive Eq. (9) from Eq. (8) are detailed in "Appendix 3" by considering the direct sound $p_{2}^{\prime}$ generated by variation in the reservoir pressure $p_{1}^{\prime}$.

\section{Experimental setups and procedure}

\subsection{Acoustic measurements setup}

In Fig. 1 a sketch of the acoustic-measurement setup is shown. A stationary non-swirling axial base flow, from left to right in Fig. 1, was created by imposing a mass-flow rate of $\dot{m}^{*}=43.0 \mathrm{~kg} \mathrm{~h}^{-1}$ in the settling chamber. This was done using a Bronckhorst F-203AV linear resistance flow controller, connected to a compressed-air supply outlet at 13 bar absolute. At this mass-flow rate, choked nozzle conditions were obtained with a reservoir pressure $p_{1}=1.12$ bar. This 
imposed an upstream nominal nozzle inlet Mach number of $M_{1}=3.67 \times 10^{-2}$. The reservoir pressure $p_{1}$ in the reservoir $V_{\text {set }}$ is measured relative to the atmospheric pressure $p_{\text {atm }}$ by means of a MKS Baratron 220D-26159 1000 mBar manometer. $p_{1}$ is, within the measurement precision, also the steady pressure in the upstream pipe connecting the reservoir to the nozzle inlet. The pressure $p_{2}$ in the section downstream from the nozzle was atmospheric $p_{\text {atm }}=1.01$ bar where the Mach number was $M_{2}=2.27 \times 10^{-2}$.

The upstream part of the setup consisted of a settling chamber $\left(V_{\text {set }}=10.5 \times 10^{-3} \mathrm{~m}^{3}\right)$ with a bell-mouth inlet to a tube section (Fig. 1). This 220-mm-long tube section had a $R_{1}=15 \mathrm{~mm}$ radius. A single tangential-injection port module (Fig. 3) was connected to the downstream end of the aforementioned tube section. The injection port module was composed of a 70-mm-long upstream pipe section with a radius of $R_{1}=15 \mathrm{~mm}$.

Unsteady tangential injection of air into the stationary axial base flow was performed through a port machined in the middle of the module, which had a small converging nozzle of outlet radius of either $R_{\theta}=1.25 \mathrm{~mm}$ or 1.50 $\mathrm{mm}$. In the following results obtained with $R_{\theta}=1.25 \mathrm{~mm}$ or $R_{\theta}=1.50 \mathrm{~mm}$ are labeled accordingly. Air injection was performed using a fast-switching valve for a variable duration of $\tau_{\theta}$. This was repeated 100 times every $3 \mathrm{~s}$. The valve was connected to the converging injection nozzle of diameter $2 R_{\theta}$ through a $37-\mathrm{mm}$-long tube with a $4 \mathrm{~mm}$ inner diameter. The injection valve was connected to a $V_{\theta}=2.8 \times 10^{-3} \mathrm{~m}^{3}$ injection reservoir, by means of a 150 -mm-long plastic hose with an inner diameter of $12 \mathrm{~mm}$. The injection reservoir was put under a pressure $p_{\theta}$, by means of a compressed-air supply system, connected to the reservoir through a $3.5-\mathrm{m}-$ long, $12-\mathrm{mm}$ inner-diameter hose. $p_{\theta}$ was set by means of a valve with a mechanical dial. The dial values were calibrated

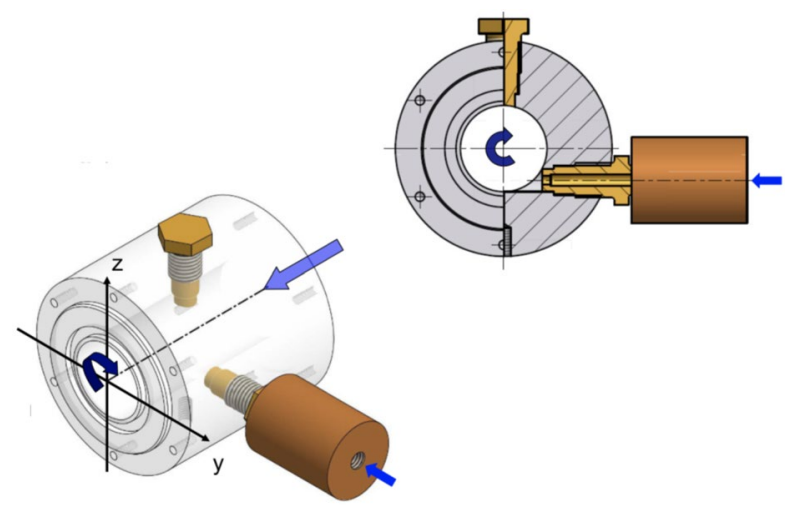

Fig. 3 The injection module with the tangential injection and radial injection ports. As was the case for tangential injection experiments, the latter is shown sealed. Air was injected at a right angle to axial flow a posteriori using a NetScanner ${ }^{T M}$ System Model 9116 manometer.

Design details of the injection valve are reported by Neuhaus and Röhle (2006), and how it was operated by Kings and Kings and Bake (2010). The nominal opening and closing times of the valve were reported by the manufacturer to be $2.5 \mathrm{~ms}$ (Neuhaus and Röhle 2006). However, the analysis of acoustic measurements in Sect. 4.2 will show that in practice, the valve-opening time is smaller. The reported $2.5 \mathrm{~ms}$ correspond to the delay between the electrical trigger signal and the injection valve actually opening. Analysis of mass-flow rate calibration measurements reported by Hirschberg et al (2021a), concisely summarized in "Appendix 2", shows that the effective radius of the choked valve was $0.948 \mathrm{~mm}$. In "Appendix 2", it is shown that for $p_{\theta}>2.5 \mathrm{bar}$, the valve was choked and the mass-flow rate was independent of the injection nozzle diameter $2 R_{\theta}$. The area of the injection ports of the choked fast-switching valve determined the impulsively injected mass-flow rate. Most of the data reported here was obtained with choked injection valve conditions.

The tangential injection module was followed downstream by a $50-\mathrm{mm}$-long uniform tube of radius $R_{1}=15 \mathrm{~mm}$. This tube was connected to a convergingdiverging nozzle with throat radius $R_{\mathrm{th}}=3.25 \mathrm{~mm}$ (crosssectional surface contraction ratio 1/16). The distance between the tangential-injection port and the nozzle inlet was $85 \mathrm{~mm}$. Downstream from the conical divergent part of nozzle (itself $250 \mathrm{~mm}$ long) was a uniform tube with a radius of $20 \mathrm{~mm}$ and a length of $1020 \mathrm{~mm}$ referred to as the "microphone section."

A GRAS 40BP 1/4" ext. polarized pressure microphone was mounted flush in its walls, calibrated using a Brüel \& Kjaer model 4228 pistonphone with $\left|p_{\text {ref }}^{\prime}\right|=123.92 \mathrm{~dB}$ and $f_{\text {ref }}=251.2 \mathrm{~Hz}$, at a distance $1150 \mathrm{~mm}$ from the nozzle throat. This was used to detect pressure waves generated by swirl-nozzle interaction. The corresponding acoustic signal $p_{2}^{\prime}$ was recorded using an OROS OR-36 12-channel analyzer with NVGate data acquisition system software, at a sampling frequency of $f_{s}=16384 \mathrm{~Hz}$.

The 1020-mm-long microphone section was connected downstream to a 24-m-long flexible tube of radius $R_{2}=20 \mathrm{~mm}$. This is a significant improvement, as it prolonged the back-and-forth travel time of acoustic waves from the microphone to the downstream open-pipe termination. This enabled the measurement of the acoustic signal $p_{2}^{\prime}$ due to unsteady swirl-nozzle interaction at the downstream microphone, without the influence of any acoustic reflections for a period of $150 \mathrm{~ms}$. Note that the effective observation time for a constant signal was restricted by the limited low-frequency response of the microphone to about $20 \mathrm{~ms}$ (Sect. 4.2). According to the 
specifications of the microphone, the deviation in measured amplitude for a harmonic signal of $10 \mathrm{~Hz}$ is $1 \mathrm{~dB}$. This corresponds to a $12 \%$ deviation in amplitude after 25 $\mathrm{ms}$ for a signal increasing linearly with time. That said, the recorded signals are sufficient to preform quantitative analysis of the rapid decrease and increase $\Delta p_{2}^{\prime}$ in pressure observed upon ingestion and evacuation of swirl.

\subsection{Steady-state flow measurements}

Steady-state measurements were obtained by replacing the fast injection valve and convergent nozzle described in Sect. 3.1, by a convergent tangential injection nozzle with injection port radius of either $R_{\theta}=1.25 \mathrm{~mm}$ or $R_{\theta}=1.50 \mathrm{~mm}$. At a $4.0 \mathrm{~mm}$ distance upstream from the tangential injection port, the radius was enlarged to $3.25 \mathrm{~mm}$. Thus, for $R_{\theta}=1.25 \mathrm{~mm}$ or $R_{\theta}=1.50 \mathrm{~mm}$ one had a contraction ratio of 0.148 or 0.213 , respectively.

The convergent tangential injection nozzle was connected to a $12-\mathrm{mm}$-inner-diameter and 1.5-m-long hose leading to an upstream installed Bronckhorst F-203AV linear-resistance flow controller. The flow controller was used to fix the steady-state tangential-injection mass-flow rate $\dot{m}_{\theta, \mathrm{st}}$. It was connected by means of a 12 -mm-innerdiameter and 1-m-long hose to a compressed air supply outlet at 13 bar absolute. This compressed air supply was the same as the one used to impose a steady axial mass-flow rate $\dot{m}$ (controlled by a separate Bronckhorst F-203AV linear resistance flow controller). Thus, the steady axial mass-flow rate $\dot{m}$ and $\dot{m}_{\theta, \text { st }}$ could be fixed separately.

The total mass-flow rate $\dot{m}_{\text {tot }}=\dot{m}+\dot{m}_{\theta, \text { st }}$ was fixed at either: $41 \mathrm{~kg} \mathrm{~h}^{-1}, 42 \mathrm{~kg} \mathrm{~h}^{-1}, 43 \mathrm{~kg} \mathrm{~h}^{-1}, 44 \mathrm{~kg} \mathrm{~h}^{-1}$ or $45 \mathrm{~kg} \mathrm{~h}^{-1}$. Keeping $\dot{m}_{\text {tot }}$ constant, $\dot{m}_{\theta, \text { st }}$ was varied between 0 and $18 \mathrm{~kg} \mathrm{~h}^{-1}$. For each setting of $\dot{m}_{\theta, \mathrm{st}}$ the reservoir pressure upstream from the choked nozzle $p_{1}$ was measured after a time lapse of five minutes (to ensure that a steady state was attained).

A multi-variable fit of the steady-state data was performed using MATLAB's "fit" function (Curve Fitting Toolbox). $\dot{m}_{\text {tot }}$ was used as the dependent variable. $p_{1}$ and $\left(\dot{m}_{\theta, \mathrm{st}} / \dot{m}\right)^{2}$ were used as the independent variables. By specifying "Poly14" in MATLAB's "fit" function; $p_{1}$ and $\left(\dot{m}_{\theta, \mathrm{st}} / \dot{m}\right)^{2}$ were fitted in the first and fourth order, respectively. The resulting correlation $\dot{m}_{\mathrm{fit}}\left(p_{1},\left(\dot{m}_{\theta, \mathrm{st}} / \dot{m}\right)^{2}\right)$ was used for the empirical quasi-steady model (Eq. (9)) proposed in Sect. 2.1.

In Sect. 4.1, the results of the multi-variable fit of the steady-state data are discussed. The resulting empirical quasi-steady model (Eq. (9)) is compared to acoustic measurement results in Sect. 4.2.3.

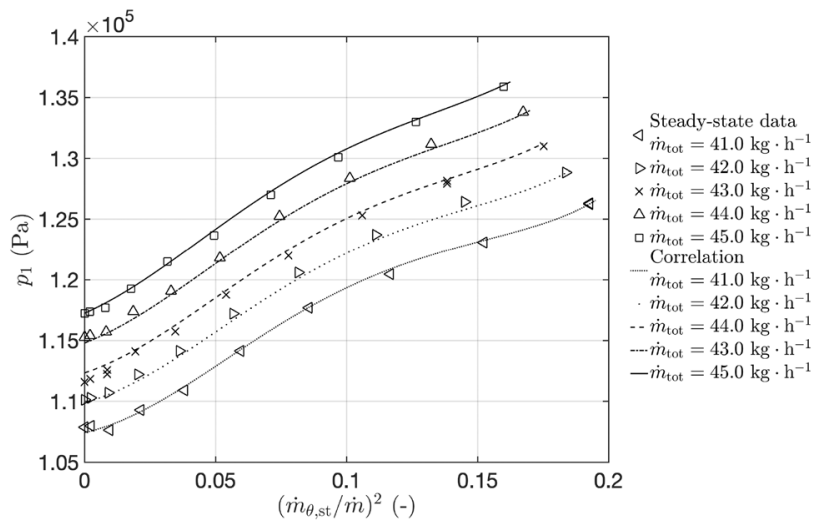

(a) $R_{\theta}=1.25 \mathrm{~mm}$

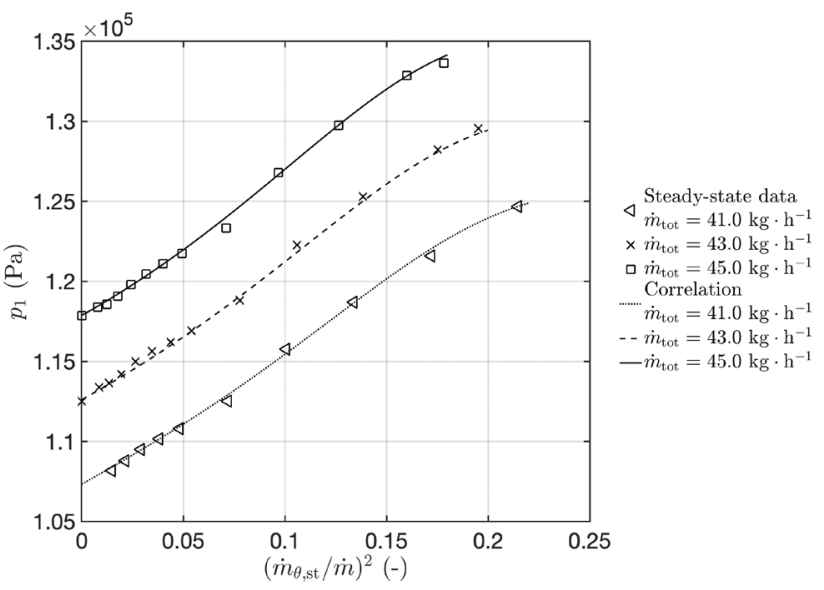

(b) $R_{\theta}=1.50 \mathrm{~mm}$

Fig. 4 Visual evidence of the good quality of the correlation obtained for steady-state measurements with: $\mathbf{a} R_{\theta}=1.25 \mathrm{~mm}$ and $\mathbf{b}$ $R_{\theta}=1.5 \mathrm{~mm}$, respectively

\section{Results}

\subsection{Steady-state results}

In this section, visual evidence is provided of the quality of the correlation $\dot{m}_{\mathrm{fit}}\left(p_{1},\left(\dot{m}_{\theta, \mathrm{st}} / \dot{m}\right)^{2}\right)$, found by applying the procedure described in Sect. 3.2. To this end, $p_{1}$ is plotted as a function of $\left(\dot{m}_{\theta, \mathrm{st}} / \dot{m}\right)^{2}$ in Fig. $4 \mathrm{a}, \mathrm{b}$ for $R_{\theta}=1.25 \mathrm{~mm}$ and $1.50 \mathrm{~mm}$, respectively. In Fig. 4, the steady-state data obtained with $\dot{m}_{\text {tot }}=41.0 \mathrm{~kg} \mathrm{~h}^{-1}, 42.0 \mathrm{~kg} \mathrm{~h}^{-1}, 43.0 \mathrm{~kg} \mathrm{~h}^{-1}$, $44.0 \mathrm{~kg} \mathrm{~h}^{-1}, 45.0 \mathrm{~kg} \mathrm{~h}^{-1}$ are indicated with left-pointing triangles, right-pointing triangles, crosses, up-pointing triangles, and squares, respectively. The correlations obtained for the different $\dot{m}_{\text {tot }}$ were used to trace lines through the steady-state data. This yields visual evidence of the good quality of $\dot{m}_{\mathrm{fit}}\left(p_{1},\left(\dot{m}_{\theta, \mathrm{st}} / \dot{m}\right)^{2}\right)$. For $\left(\dot{m}_{\theta} / \dot{m}\right)^{2}<0.03$ the deviation between experimental values of $p_{1}$ and the fit becomes significant compared to the effect of the tangential 
injection on $p_{1}$. Hence, the fit is not reliable for such low $\left(\dot{m}_{\theta} / \dot{m}\right)^{2}$. The $\dot{m}_{\text {tot }}=43.0 \mathrm{~kg} \mathrm{~h}^{-1}$ correlation was used for the quasi-steady model Eq. (9) (proposed in Sect. 2.2). This quasi-steady model was applied and compared to acoustic measurement data as reported in Sect. 4.2. Note that as predicted by the theory described in "Appendix 1", for a specified ratio $\dot{m}_{\theta} / \dot{m}, \dot{m}$ is a linear function of $p_{1}$. Additionally, these measurements indicate that the steady massflow rate in the absence of tangential injection $\left(\dot{m}_{\theta}=0\right)$ is well predicted by a quasi-one dimensional model; one has $\dot{m}_{\mathrm{fit}}\left(p_{1}, 0\right)=(1.020 \pm 0.005) \dot{m}^{*}$.

Note that as $\left(\dot{m}_{\theta} / \dot{m}\right)^{2}$ is increased, $p_{1}$ is systematically larger for $R_{\theta}=1.25 \mathrm{~mm}$ than for $1.50 \mathrm{~mm}$. This indicates a higher swirl for the data obtained with $R_{\theta}=1.25 \mathrm{~mm}$. Thus,
$R_{\theta}$ influences $\Delta m_{\mathrm{st}}$. This indicates that the swirl generated by tangential injection is a function of both the mass-flux $\dot{m}_{\theta}$ and the injection-port radius $R_{\theta}$.

\subsection{Acoustic measurement results}

\subsubsection{A typical experiment}

The acoustic signals, recorded as a result of a 100 consecutive $3 \mathrm{~s}$ measurements, were phase averaged using the technique reported by Kings and Bake 2010. The phase-averaged signal contained strong acoustic oscillations primarily due to a quarter-wavelength oscillation (ca. $250 \mathrm{~Hz}$ ) of the upstream pipe section of the setup (Hirschberg et al 2021b).
Fig. 5 a Upstream recorded reservoir pressure change $p_{1}^{\prime}$ due to tangential injection. $\mathbf{b}$ Downstream recorded acoustic signal $p_{2}^{\prime}$. These results were obtained with $p_{\theta}=5.27 \mathrm{bar}$ and $R_{\theta}=1.25 \mathrm{~mm}$. The injection port was located $85 \mathrm{~mm}$ upstream from the nozzle inlet. The vertical lines indicate key events, such as, e.g., the opening and closing of the valve. On the right-hand vertical axis the rectangular-electrical pulse $V_{\text {valve }}$ with a $10 \mathrm{~V}$ and $10 \mathrm{~ms}$ width used for the opening and closing of the fast-switching valve is indicated by a blue line (color online)

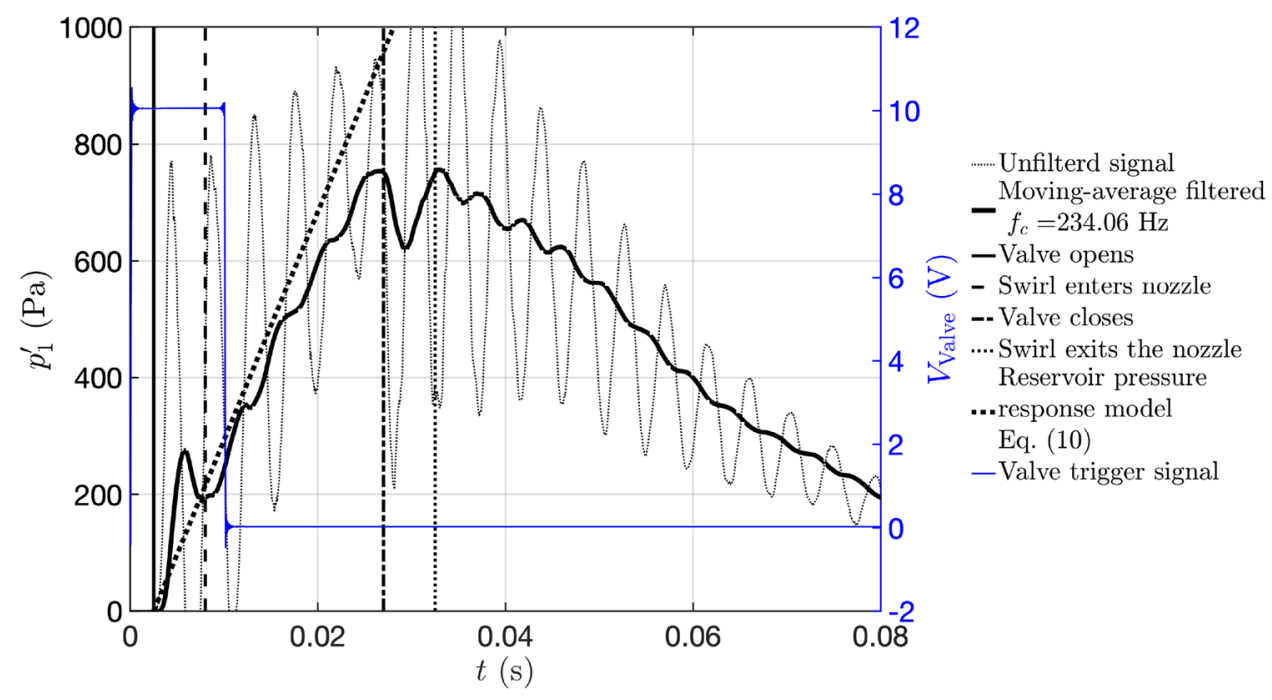

(a) Upstream microphone.

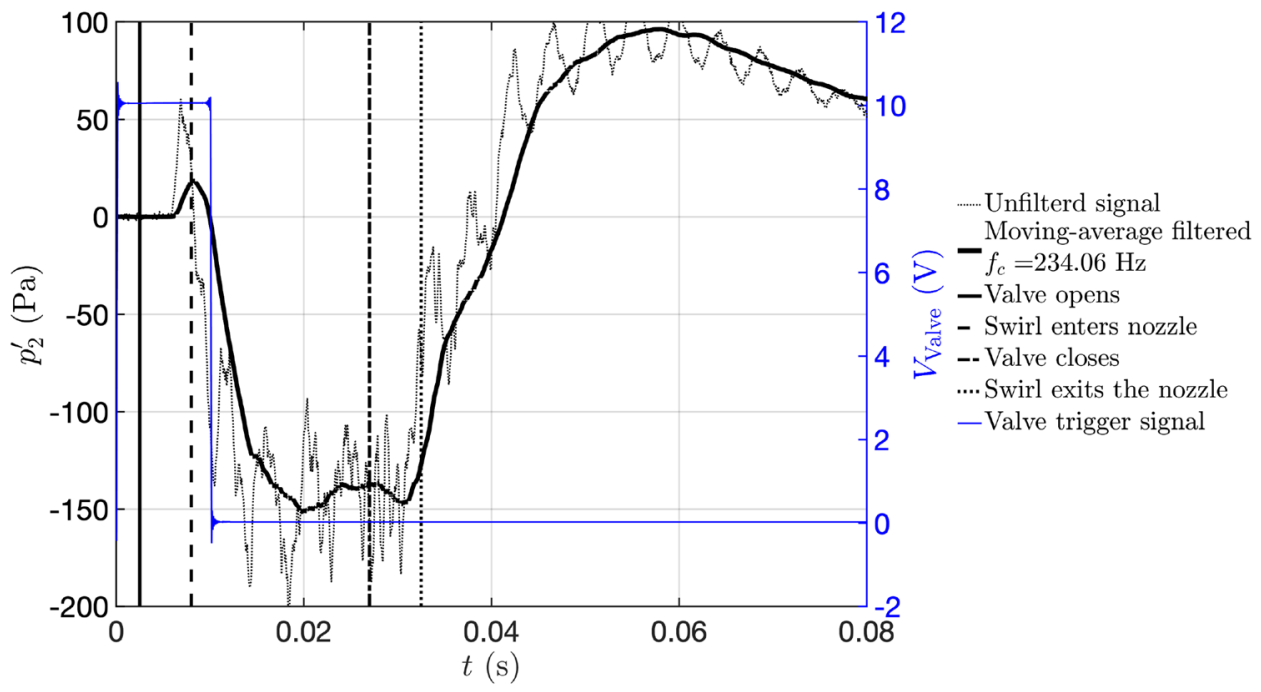

(b) Downstream microphone. 
These spurious oscillations can clearly be seen in the nonfiltered acoustic signals (finely dashed black lines) in Fig. 5.

The results shown in Fig. 5 were obtained with a $10 \mathrm{~ms}$ long square pulse of $10 \mathrm{~V}$ amplitude (blue line and vertical axis on the right-hand side (color online)), used to trigger the opening and closing of the valve. The absolute injection-reservoir pressure $p_{\theta}$ was 5.27 bar. The vertical axes, in Fig. 5a, b correspond to the upstream $p_{1}^{\prime}$ (microphone positioned $270 \mathrm{~mm}$ upstream from the nozzle inlet) and downstream $p_{2}^{\prime}$ (microphone positioned $1150 \mathrm{~mm}$ downstream from the nozzle throat) acoustic signals. In both, the finely dotted lines correspond to the phase-averaged signals, while the solid black lines correspond to these signals filtered using a moving-average filter with a low-pass cut-off frequency of $f_{c}=234.06 \mathrm{~Hz}$. Note that the moving-average filter causes a $2 \mathrm{~ms}$ delay of the signal. The solid vertical lines at $t=2.5 \mathrm{~ms}$ correspond to the point in time when the tangential-injection valve was opened. The vertical dashed lines at $8 \mathrm{~ms}$ correspond to the approximate moment the upstream generated swirl structure entered the nozzle. At this point the presence of swirl decreased the mass-flow rate through the choked nozzle, which caused a negative signal to be emitted downstream. This swirl-nozzle interaction signal had an amplitude of ca. $\Delta p_{2}^{\prime}=-150 \mathrm{~Pa}$, as can clearly be seen in Fig. 5b. The acoustic pressure $p_{2}^{\prime}$ remains fairly constant until $t=30 \mathrm{~ms}$, about $5.5 \mathrm{~ms}$ after the vertical dasheddotted line corresponding to the moment when the valve was closed. One notes that $t=27 \mathrm{~ms}$ corresponds to the point in time were the upstream reservoir pressure $p_{1}$ ceased to rise, as can be seen in Fig. 5a. The dotted lines at $t=32.5 \mathrm{~ms}$ correspond to the approximate moment at which the swirl exits the nozzle. Without the presence of swirl in the nozzle throat, the mass-flow rate returns back to its swirl-free steady state value, causing $p_{2}^{\prime}$ to increase.

Although the valve had a reported opening time of $2.5 \mathrm{~ms}$, the authors found that the pressure response, measured by means of a GRAS 40BP 1/4" ext. polarized pressure microphone mounted flush in the wall $185 \mathrm{~mm}$ upstream from the tangential-injection point, showed a delay between the electronic trigger for opening and the valve opening (Fig. 5a). The actual effective valve-opening time was very short, estimated to be of the order of $1.0 \mathrm{~ms}$. For closing, the delay time between the electrical signal and the closing of the valve was of the order of $10 \mathrm{~ms}$ decreasing with increasing $p_{\theta}$. Again, as for the opening, the actual closing time was much shorter than the delay time.

In "Appendix 3", the following model for the increase in upstream reservoir pressure $p_{1}$ is constructed

$p_{1}^{\prime}=\frac{\mathrm{d} p_{1}^{\prime}}{\mathrm{d} t}\left(t-t_{\text {open }}\right) \simeq \frac{c_{1}^{2} \dot{m}_{\theta}}{V_{\text {set }}}\left(t-t_{\text {open }}\right)$ were $t_{\text {open }}=2.5 \mathrm{~ms}$ is the moment the tangential-injection valve is opened. The assumptions that the change in $\dot{m}$ is negligible and that $\dot{m}_{\theta}$ is constant imply the predicted linear increase in pressure shown as a thick dotted black line in Fig. 5a. One observes that Eq. (10) is fairly accurate in predicting the initial linear increase in upstream reservoir pressure due to tangential air injection. This underpins the validity of the static calibration, described in "Appendix 2". At $t=25 \mathrm{~ms}$, just before the closing of the valve, one observes a $15 \%$ deviation of the upstream pressure from the predicted linear increase. The authors assume that this to be due to the limited low-frequency response of the microphone. Indeed, the microphone specifications mention a deviation of $1 \mathrm{~dB}$ at $10 \mathrm{~Hz}$ in the response to a harmonic signal. This is equivalent to a $12 \%$ deviation after a linear rise during $25 \mathrm{~ms}$ (a quarter period of oscillation).

As the valve closes, $p_{1}^{\prime}$ starts to decrease. The time constant for the decrease of the reservoir pressure perturbation is ca. $1.0 \mathrm{~s}$ ("Appendix 3"). Hence, the $p_{1}$ decay from $t=26$ $\mathrm{ms}$ to $t=80 \mathrm{~ms}$ should be ca. $5.4 \%$ of the maximum pressure $\left|\Delta p_{1}^{\prime}\right|_{\max }=770 \mathrm{~Pa}$ reached at $t=26 \mathrm{~ms}$. However, a much faster decrease of the signal is observed after $t=40 \mathrm{~ms}$. This confirms the limited low frequency response of the microphone.

The upstream pressure fluctuations $p_{1}^{\prime}$ are partially transmitted to the downstream microphone as so-called direct sound (Kings and Bake 2010; Hirschberg et al 2021b). This causes the initial positive pressure pulse observed in Fig. 5a around $t=7 \mathrm{~ms}$. The effect of the gradual increase of $p_{1}$ on $p_{2}^{\prime}$ is less obvious. For high upstream unsteady injection mass-flow rates $\dot{m}_{\theta}$ it appears to be negligible, because $p_{1}$ increases linearly with $\dot{m}_{\theta}$ while $p_{2}^{\prime}$ is proportional to $\dot{m}_{\theta}^{2}$. This was confirmed by experiments in which the upstream air injection was radial. The results of these radial injection experiments are analyzed in "Appendix 3". For the experimental signal displayed in Fig. $5 \mathrm{~b}$ at $t=30 \mathrm{~ms}$, the direct sound due to the slow increase in $p_{1}$ results in an increase of ca. $15 \mathrm{~Pa}$ relative to the lowest value $\left(\Delta p_{2}^{\prime}\right)_{\max }=-150 \mathrm{~Pa}$ reached at $t=20 \mathrm{~ms}$. Accounting for this effect, one concludes that $p_{2}^{\prime}$ has reached a plateau at $t \simeq 20 \mathrm{~ms}$. The pressure remains constant until the swirl is evacuated, which occurs at $t \simeq 32.5 \mathrm{~ms}$. The gradual increase of $p_{1}$ has, relatively speaking, a more significant effect on $p_{2}^{\prime}$ for lower unsteady mass-flow rates. In this text, the data have not been corrected for the influence of direct sound, as such a correction would not change the conclusions drawn from the data.

\subsubsection{Variation of the tangential-injection time}

As was explained above, the results in Fig. 5b show that within $5 \mathrm{~ms}$ after the start of the decrease in downstream pressure $p_{2}^{\prime}$, a plateau is reached at $p_{2}^{\prime} \simeq-150 \mathrm{~Pa}$. This plateau indicates quasi-steady behavior (Hirschberg et al 


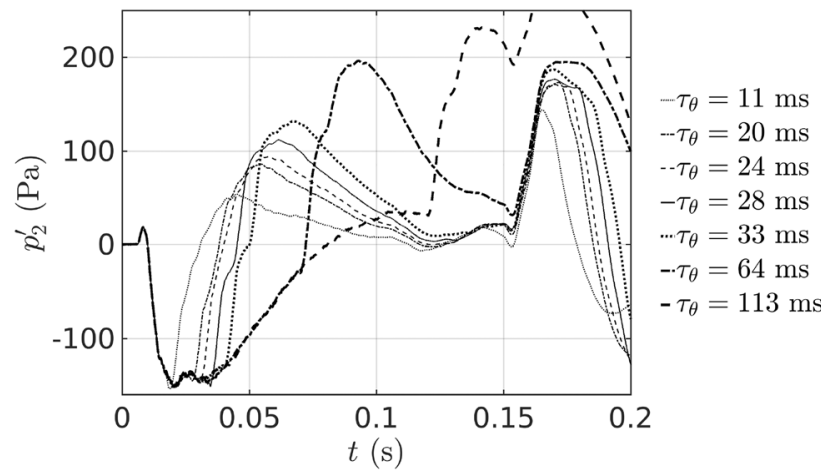

Fig. 6 Moving-average filtered $\left(f_{c}=234.06 \mathrm{~Hz}\right)$ downstreammeasured acoustic response $p_{2}^{\prime}$ due to swirl-nozzle interaction, for seven $\tau_{\theta}$ values. These results were obtained with $R_{\theta}=1.25 \mathrm{~mm}$ and $p_{\theta}=5.2$ bar. The injection port was located $85 \mathrm{~mm}$ upstream from the nozzle inlet

2021a). In Fig. 6, results are shown which were obtained with electrical pulse widths of $3.0 \mathrm{~ms}, 7.0 \mathrm{~ms}, 14 \mathrm{~ms}$, $20 \mathrm{~ms}, 50 \mathrm{~ms}$, and $100 \mathrm{~ms}$. These pulse widths correspond to $\tau_{\theta} \simeq 11 \mathrm{~ms}$ (the shortest experimentally achievable; dotted line), $\tau_{\theta} \simeq 20 \mathrm{~ms}$ (dashed-dotted line), $\tau_{\theta} \simeq 24 \mathrm{~ms}$ (dashed line), and $\tau_{\theta} \simeq 28 \mathrm{~ms}$ (solid line), $\tau_{\theta} \simeq 33 \mathrm{~ms}$ (thick dotted line), $\tau_{\theta} \simeq 64 \mathrm{~ms}$ (thick dashed-dotted line), $\tau_{\theta} \simeq 113 \mathrm{~ms}$ (thick dashed line), respectively.

Note that the time it takes for an plane acoustic wave to travel from the downstream microphone to the downstream termination and back is ca. $144 \mathrm{~ms}$. Thus, in Fig. 6 the $p_{2}^{\prime}$ signal for $t>150 \mathrm{~ms}$ is polluted by an upstream traveling reflection of the signal emitted after $t \simeq 7.0 \mathrm{~ms}$.

In Fig. 6, one observes a negative plateau for the first 20 $\mathrm{ms}$ after the short positive direct-sound pulse at $t=10 \mathrm{~ms}$, due to the opening of the valve. When the valve closes, one observes a similar small expansion peak (negative) due to the direct sound. This is followed by a sudden increase in $p_{2}^{\prime}$. As it was for the signal due to swirl ingestion, the observed variation of signal due to the evacuation of swirl is reliable for $20 \mathrm{~ms}$ after the onset of the abrupt rise in $p_{2}^{\prime}$ due to evacuation of the swirl. This makes the determination of the total change in acoustic pressure from the moment the valve closes up to the maximum of acoustic pressure $\Delta p_{2}^{\prime}$ possible. Note that, to account for the limited low-frequency response of the microphone, the increase in signal after the maximum in $p_{2}^{\prime}$ has been reached is determined using the value of $p_{2}^{\prime}$ just before the negative direct-sound pulse as the zeroreference, which is used to determine the total increase $\Delta p_{2}^{\prime}$ resulting from the evacuation of the swirl from the nozzle.

The shortest achievable tangential-injection time was $\tau_{\theta} \simeq 11 \mathrm{~ms}$. This corresponds to an axial length, $\tau_{\theta} U_{1} \simeq 14 \mathrm{~cm}$, of the swirl structure upstream from the nozzle. One notes that this smallest axial length of the upstream swirl structure is quite large compared to the axial nozzle

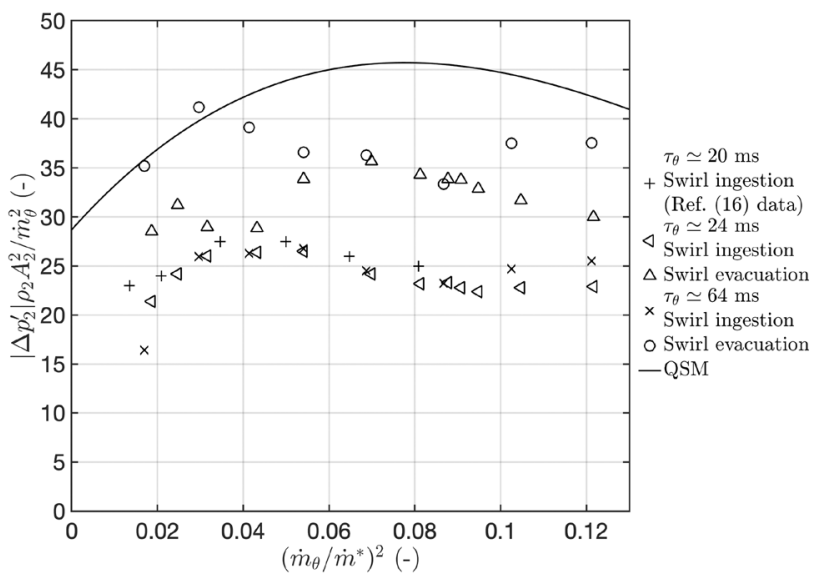

(a) $R_{\theta}=1.25 \mathrm{~mm}$

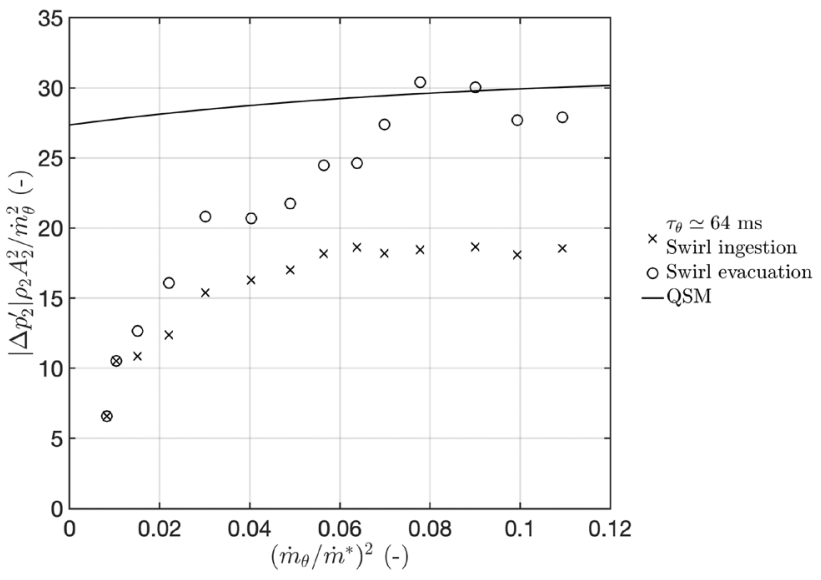

(b) $R_{\theta}=1.5 \mathrm{~mm}$

Fig. 7 The dimensionless downstream-measured acoustic response $\left|\Delta p_{2}^{\prime}\right| \rho_{2} A_{2} / \dot{m}_{\theta}^{2}$ as a function of $\left(\dot{m}_{\theta} / \dot{m}^{*}\right)^{2}$. Measurement data are compared to the scaled empirical quasi-steady model prediction (QSM line)

length. The axial vortex is also significantly elongated as it passes through the nozzle. Both justifying the application of a quasi-steady modeling approach, as proposed in Sect. 2.2. This can be anticipated to hold for large contraction ratios or equivalently small upstream Mach numbers $\left(\mathrm{O}\left(10^{-1}\right)\right.$ or smaller) and for upstream axial vortex lengths of at least the order of the upstream pipe diameter. The quasi-steady model (Eq. (9)) is compared to acoustic measurement data in the following Sect. 4.2.3.

\subsubsection{Comparison with empirical quasi-steady model}

In Fig. 7, the acoustic response amplitude $\Delta p_{2}^{\prime}$ due to swirl being ingested or evacuated by the choked nozzle is compared to the prediction using the proposed empirical quasisteady model $\left|\Delta p_{2, \mathrm{QSM}}^{\prime}\right| \rho_{2} A_{2}^{2} / \dot{m}_{\theta}^{2}$ (QSM line obtained using 
Eq. (9)). The parameter $\dot{m}_{\theta}$ in the fit $\dot{m}_{\text {fit }}\left(p_{1},\left(\dot{m}_{\theta} / \dot{m}\right)^{2}\right)$ is determined using the steady-flow calibration described in "Appendix 2", with the static value of the injection reservoir pressure $p_{\theta}$ as input. For the mass-flow rate $\dot{m}$ the value measured before the start of the experiment was used. This is justified because $\Delta \dot{m} / \dot{m}$ is small. In Fig. $7 \mathrm{a}, \mathrm{b}$ data obtained with $R_{\theta}=1.25 \mathrm{~mm}$ and $1.5 \mathrm{~mm}$ are shown, respectively.

In Fig. 7a, data obtained for $R_{\theta}=1.25 \mathrm{~mm}$ with three values of $\tau_{\theta}$ are displayed, viz. $\tau_{\theta}=20 \mathrm{~ms}, 24 \mathrm{~ms}$ and $64 \mathrm{~ms}$. The plus-sign crosses, right-pointing triangles and crosses indicate the acoustic amplitudes determined due to swirl ingestion. Acoustic amplitudes determined due to swirl evacuation of swirl by the nozzle are indicated with upright triangles and circles. Multiplication crosses and circles were also used in Fig. 7b to indicated swirl ingestion and evacuation data for $R_{\theta}=1.50 \mathrm{~mm}$. Note, that the $\tau_{\theta}=20 \mathrm{~ms}$ data points indicated with plus-sign crosses in Fig. 7a, were taken from Hirschberg et al (2021a). Due to a significantly shorter tube section downstream from the microphone, the data of Hirschberg et al (2021a) do not contain the effect of swirl evacuation by the nozzle.

One notes that overall the QSM provides a prediction well within an order-of-magnitude for all data. Furthermore, $\Delta p_{2}^{\prime}$ was scaled by multiplying by $\rho_{2} A_{2} / \dot{m}_{\theta}^{2}$, as first suggested by Hirschberg et al (2021a), which confirms the scaling rule $\Delta p_{2}^{\prime} \propto \dot{m}_{\theta}^{2}$ proposed by Hirschberg et al (2021a).

However, one observes in Fig. 7a for $\left(\dot{m}_{\theta} / \dot{m}^{*}\right)^{2}>0.02$ and in Fig. $7 \mathrm{~b}$ for $\left(\dot{m}_{\theta} / \dot{m}^{*}\right)^{2}>0.03$, a better agreement for the swirl evacuation cases than for swirl ingestion. The authors believe that this was due to the fact that air is injected at very high speed and at a right angle to the axial flow. This creates a wall-bounded jet, which meets itself after one revolution. The authors assume that this causes a split; one part of the wall bounded jet moves downstream to the nozzle inlet, whilst the other part moves upstream. I.e., only a part of the injected angular momentum flux is initially ingested by the nozzle. The analysis of Kings' (2015) hotwire measurements reported by Hirschberg et al (2021b) provided evidence of an upstream moving central jet in these types of pipe flow. After the tangential injection valve is closed, the accumulated angular momentum in the upstream pipe section convects through the nozzle. This is believed to be the reason for the better predictive value of the QSM for $\Delta p_{2}^{\prime}$ due to evacuation of the axially oriented vortex. Further discussion is provided at the end of this section.

One notes that for $\left(\dot{m}_{\theta} / \dot{m}^{*}\right)^{2}<0.03$ there is considerable scatter in results for the swirl ingestion (Fig. 7a). This indicates that, for $\left(\dot{m}_{\theta} / \dot{m}^{*}\right)^{2}<0.03$, there is a significant uncertainty in the results. This provides an indication for the limitations of the results when drawing conclusions.

In Sect. 4.1, it was noted that there is an influence of $R_{\theta}$ on $\Delta \dot{m}_{\mathrm{st}}$ at constant tangential mass-flow rate injection $\dot{m}_{\theta}$
(Fig. 4). A similar effect is observed in Fig. 7, where the downstream dimensionless amplitude decreases systematically as $R_{\theta}$ is increased from $1.25 \mathrm{~mm}$ and $1.50 \mathrm{~mm}$. The investigation of this effect falls out of the scope of the present text.

In Fig. $8, \Delta p_{2}^{\prime} / \Delta p_{2, \mathrm{QSM}}^{\prime}$ due to swirl evacuating the choked nozzle is plotted as a function of $\tau_{\theta} U_{1} / L_{1}$, where $L_{1}=340 \mathrm{~mm}$ is the length of the pipe upstream from the nozzle, and $U_{1}=12.5 \mathrm{~m} \mathrm{~s}^{-1}$ is the average axial velocity in the upstream pipe. Data obtained with $p_{\theta}=5.3$ bar and 6.2 bar are indicated with circles and squares, respectively. All data were obtained with $R_{\theta}=1.25 \mathrm{~mm}$. The data points of the respective sets are connected by straight line segments. This was done to give the reader an impression of the global trend in the two data sets. N.b., these line segments are not the product of a statistical-data fit, nor the application of a predictive model. One observes that Eq. (9) has good predictive quality for the $p_{\theta}=5.3$ bar data. Indeed, for these data one finds the largest deviation to be $16 \%$ at $\tau_{\theta} U_{1} / L_{1}=4.15$. For the $p_{\theta}=6.2$ bar data, the largest deviation is ca. $60 \%$ at $\tau_{\theta} U_{1} / L_{1}=1.00$, which is larger than what was observed for the other data set. Still, for both data sets the overall predictive quality of the empirical quasi-steady model (Eq. (9)) is well within an order-of-magnitude.

One concludes that the time needed to approach quasisteady behavior is of the order of a few periods of length $L_{1} / U_{1}$, which is the estimated convection time from the upstream bell-mouth inlet to the nozzle inlet. One also notes that the time taken to reach the maximum $\Delta p_{2}^{\prime} / \Delta p_{2, \mathrm{QSM}}^{\prime}$ is of the order of this convection time. I.e., most of the swirl causing the downstream-measured acoustic signal is concentrated in the upstream pipe segment. Thus, the settling chamber did not have a significant impact on the swirl-nozzle interaction signal under the considered conditions.

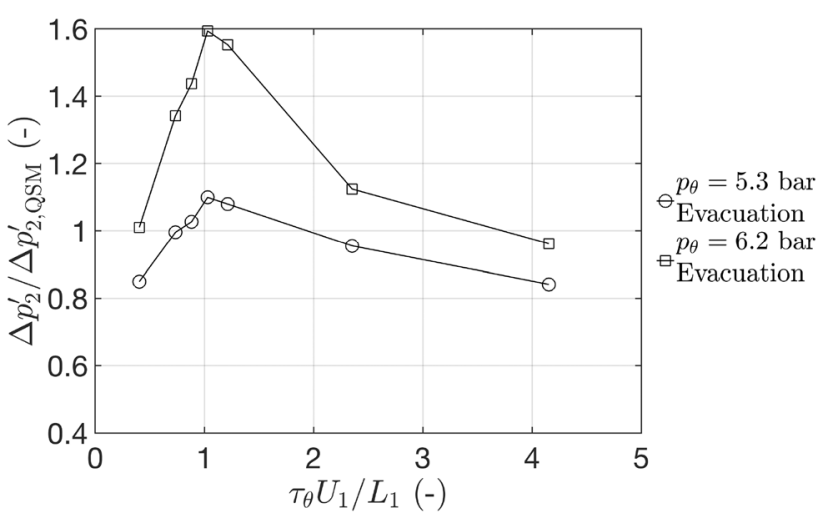

Fig. 8 Comparison of the downstream-measured acoustic amplitude $\Delta p_{2}^{\prime}$ to the empirical quasi-steady model (QSM) prediction $\Delta p_{2, \mathrm{QSM}}^{\prime}$ as a function of $\tau_{\theta} U_{1} / L_{1}$ 
The observed overshoot in $\Delta p_{2}^{\prime} / \Delta p_{2, \mathrm{QSM}}^{\prime}$ around $\tau_{\theta} U_{1} / L_{1}=1$ provides an indication of the complicated flow behavior (previously hypothesized looping wall bounded jet) upstream from the nozzle. This points to unsteady flow effects upstream from the nozzle, to be investigated in a follow up study. The authors believe that numerical simulations would be an appropriate tool for such an investigation.

\section{Conclusion}

The proposed analytical quasi-cylindrical model for swirl-nozzle interaction provides a quantitative prediction of the mass-flow rate reduction due to interaction of an axial vortex with a choked nozzle under steady-flow conditions. This model can, for low upstream Mach numbers, be applied to describe the unsteady interaction of a longitudinal vortex with a length of at least one nozzle inlet diameter. The time-dependent modulation of the mass-flow rate generates acoustic waves in the pipe downstream of the nozzle. These are a measure for the swirl of the axial vortex. The downstream propagating acoustic waves are most easily detected when a long downstream pipe is used. This makes the momentary downstream measurement of the outgoing acoustic waves possible, without the interference of upstream traveling reflections.

In first-order approximation, for a choked convergent injection nozzle the swirl generated by tangential injection is proportional to the injected mass-flow rate. This simple scaling law fails at lower tangential injection mass-flow rates. The data also indicate that the swirl decreases with increasing tangential injection nozzle diameter.

An empirical quasi-steady model based on steady-flow measurements of the reservoir pressure as a function of the tangential injection mass-flow rates was developed. This model provided good predictions for the amplitude of the downstream acoustic signal due to the evacuation of swirl confirming its validity. In contrast, the measured downstream amplitude due to ingestion of swirl by the nozzle (after the abrupt start of a tangential injection) is about half of the empirical quasi-steady model prediction. This is due to the fact that initially a large part of the tangentially injected flow is deflected upstream. Moreover, the flow due to the tangential injection is initially dominated by a thin wall-bounded jet, which must undergo a significant transition to the much more uniform velocity distribution of a steady swirling flow. This transition was observed in measurements for which the tangential-injection time was varied.

In summary, observations indicate that the downstream acoustic signal can be used as a diagnostic tool to monitor key aspects of the flow dynamics in systems with swirl introduced upstream from choked-nozzle outlets, e.g., in swirl-stabilized rocket motors.

Here cold-gas experiments were reported, viz. without combustion. Although this omits other effects, such as entropy noise, from consideration it also prevents their presence from confounding the interpretation of the results. The present study thus demonstrates that such model experiments can be used as manageable tools for exploration, as well as for the development and validation of theory.

\section{Appendix 1: Quasi-cylindrical flow approximation}

Assuming a uniform pressure in the pipe cross section $A$, one finds using the equation of Bernoulli for compressible flow:

$U \frac{\mathrm{d} U}{\mathrm{~d} x}=-\frac{1}{\rho} \frac{\mathrm{d} p}{\mathrm{~d} x}$

where for an axisymmetric flow one has $U^{2}=u_{x}^{2}+u_{\theta}^{2}$. The flow is assumed isentropic, as such:

$\frac{\mathrm{d} p}{\mathrm{~d} x}=c^{2} \frac{\mathrm{d} \rho}{\mathrm{d} x}$.

The conservation of mass in differential form is:

$\frac{1}{\rho} \frac{\mathrm{d} \rho}{\mathrm{d} x}+\frac{1}{u_{x}} \frac{\mathrm{d} u_{x}}{\mathrm{~d} x}+\frac{1}{A} \frac{\mathrm{d} A}{\mathrm{~d} x}=0$

The integral conservation of angular momentum

$\int_{0}^{R} \rho u_{\theta} u_{x} r^{2} \mathrm{~d} r=\mathrm{constant}$

combined with the mass conservation (Eq.(13)) for uniform velocities $u_{x}, u_{\theta}$ and density $\rho$ in a cross section, yields

$\frac{1}{u_{\theta}} \frac{\mathrm{d} u_{\theta}}{\mathrm{d} x}+\frac{1}{2 A} \frac{\mathrm{d} A}{\mathrm{~d} x}=0$

This equation is equivalent to the conservation of the circulation $\Gamma=2 \pi R u_{\theta}$ in a frictionless pipe flow.

After some algebra, one finds:

$u_{x} \frac{\mathrm{d} u_{x}}{\mathrm{~d} x}\left(1-\frac{c^{2}}{u_{x}^{2}}\right)=\frac{c^{2}}{A} \frac{\mathrm{d} A}{\mathrm{~d} x}\left(1+\frac{1}{2} \frac{u_{\theta}^{2}}{c^{2}}\right)$

At the throat, one has $A=A_{\mathrm{th}}$ and $\mathrm{d} A / \mathrm{d} x=0$, the latter and Eq. (16) imply, $u_{x, \text { th }}=c_{\text {th }}$ for choked-nozzle flow. One notes that this results is a sonic condition analogous to the one for choked-nozzle flows without the presence of swirl.

Because an ideal gas $p=\rho R_{\mathrm{spf}} T$ with constant specific heat is assumed, one has 


$$
\frac{p}{p_{1}}=\left(\frac{\rho}{\rho_{1}}\right)^{\gamma} .
$$

The steady-state mass-flow rate becomes

$\dot{m}_{\mathrm{st}}=\rho_{\mathrm{th}} c_{\mathrm{th}} A_{\mathrm{th}}=\rho^{*} c^{*} A_{\mathrm{th}}\left(\frac{T_{\mathrm{th}}}{T^{*}}\right)^{\frac{\gamma+1}{2(\gamma-1)}}$

Using the Bernoulli equation, one finds

$\frac{T^{*}}{T_{\mathrm{th}}}=\left(\frac{c^{*}}{c_{\mathrm{th}}}\right)^{2}=\frac{1+\frac{\gamma-1}{2}\left(\left(\frac{u_{\mathrm{x}, \mathrm{hh}}}{c_{\mathrm{th}}}\right)^{2}+\left(\frac{u_{\theta \mathrm{th}}}{c_{\mathrm{th}}}\right)^{2}\right)}{1+\frac{\gamma-1}{2}}$

Exploiting the choking condition $u_{x, \mathrm{th}}=c_{\mathrm{th}}$, one finds

$\frac{T^{*}}{T_{\mathrm{th}}}=\frac{1}{1-\frac{\gamma-1}{\gamma+1}\left(\frac{u_{\theta, \mathrm{th}}}{c^{*}}\right)^{2}}$.

Defining $\Delta \dot{m}_{\mathrm{st}} / \dot{m}^{*} \equiv\left(\dot{m}_{\mathrm{st}}-\dot{m}^{*}\right) / \dot{m}^{*}$, one obtains:

$\frac{\Delta \dot{m}_{\mathrm{st}}}{\dot{m}^{*}}=\left(1-\frac{\gamma-1}{\gamma+1}\left(\frac{u_{\theta, \mathrm{th}}}{c^{*}}\right)^{2}\right)^{\frac{\gamma+1}{2(\gamma-1)}}-1$.

Note that because a uniform pressure was assumed in a cross section, this result is only valid for $\left(u_{\theta, \mathrm{th}} / c^{*}\right)^{2}<<1$. Therefore, the excellent agreement up to $\left(u_{\theta, \mathrm{th}} / c^{*}\right)^{2}=0.25$ of this model with the experiments and numerical results reported by Dutton (1989) (as shown in Fig. 2) is pleasantly surprising.

\section{Appendix 2: Calibration of the unsteady tangential mass-flow rate}

The unsteady tangential injection mass-flow rate $\dot{m}_{\theta}$ was determined from the measured tangential injection reservoir pressure $p_{\theta}$. To determine $\dot{m}_{\theta}$ as a function of the injection reservoir pressure $p_{\theta}$, steady-flow measurements were used. For this purpose, a Bronckhorst F-203AV linear resistance flow meter was installed upstream of the tangential injection reservoir. The axial injection $\dot{m}_{1}$ was set to zero. I.e., during the calibration procedure $p_{1}=p_{2}=p_{\mathrm{atm}}$. This was done to ensure that a $60 \mathrm{~s}$ tangential injection would not cause a dangerous increase of pressure in the setup (Fig. 1). A $60 \mathrm{~s}$ tangential injection time was necessary to ensure that the Bronckhorst flow meter measured $\dot{m}_{\theta}$ reliably. $p_{\theta}$ was measured using a NetScanner ${ }^{T M}$ System Model 9116 manometer, after $55 \mathrm{~s}$ of tangential injection. Calibration was obtained for both tangential injection outlet port radius $R_{\theta}=1.25 \mathrm{~mm}$ and $1.50 \mathrm{~mm}$. The temperature in the laboratory was measured, and found to be $T_{\mathrm{atm}}=293.9 \mathrm{~K}$.

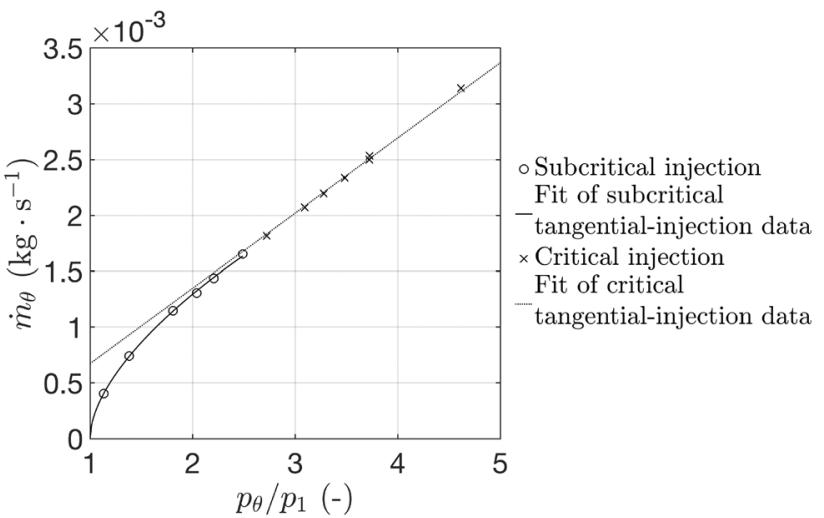

Fig. $9 \dot{m}_{\theta}$ as a function of $p_{\theta} / p_{1}$, for $R_{\theta}=1.25 \mathrm{~mm}$

In Fig. 9, the tangential injection mass-flow rate $\dot{m}_{\theta}$ is plotted as a function of $p_{\theta} / p_{1}$, in which the two regimes are visible. The first (open circles) obtained with subcritical flow at the valve $\left(p_{\theta} / p_{1}<2.0\right)$, and the second (crosses) obtained with critical tangential injection $\left(p_{\theta} / p_{1}>2.5\right)$, i.e., a choked valve flow. For flow conditions at which the valve is choked, the critical injection radius was determined to be $R^{*}=0.948 \mathrm{~mm}$ (Hirschberg et al 2021a). For the subcritical tangential-injection data $p_{\theta} / p_{1}<2.0$, the following fit is proposed

$\dot{m}_{\theta}=\rho_{1} c_{1}\left(\frac{p_{\theta}}{p_{1}}\right)^{(\gamma-1) /(2 \gamma)} M_{\mathrm{inj}} S_{j}$

where $S_{j}$ and $M_{\text {inj }}$ are the effective injection jet cross section and injection Mach number, respectively. $S_{j}$ is calculated using the following experimental fit relation

$S_{j}=\pi\left(L_{1} M_{\text {inj }}+L_{0}\right)^{2}$

where $L_{0}=0.79313 \mathrm{~mm}$ and $L_{1}=0.13027 \mathrm{~mm}$ for $R_{\theta}=1.25 \mathrm{~mm}$; for $R_{\theta}=1.50 \mathrm{~mm} L_{0}=0.86662 \mathrm{~mm}$ and $L_{1}=0.077090 \mathrm{~mm}$. The effective jet Mach number $M_{\mathrm{inj}}$ is determined using

$M_{\mathrm{inj}}=\sqrt{\frac{2}{\gamma-1}\left(\left(\frac{p_{\theta}}{p_{1}}\right)^{(\gamma-1) / \gamma}-1\right)}$.

Equation (22) is based on a compressible isentropic flow model between the valve and the injection jet. Additional losses occur downstream from the valve, and upstream of the injection port. Therefore, the effective injection Mach number $M_{\text {inj }}$ is not an actual jet Mach number and can be larger than unity. 


\section{Appendix 3: Direct sound due to slow change in reservoir conditions}

To analyze the long-term increase of the reservoir pressure $p_{1}$ due to unsteady tangential injection, an integral mass balance is used. It is assumed that compression due to unsteady tangential injection is adiabatic and occurs uniformly in the upstream reservoir. I.e., the quarter wavelength oscillation in the upstream pipe segment is ignored. Considering a linear model in $p_{1}^{\prime}$, one neglects possible changes in mass-flow rate $\dot{m}$ through the convergent-divergent nozzle to find:

$\frac{V_{\text {set }}}{c_{1}^{2}} \frac{\mathrm{d} p_{1}^{\prime}}{\mathrm{d} t}=\dot{m}_{\theta}$.

$\dot{m}_{\theta}$ is assumed constant and to corresponds to the static calibration for given constant pressure $p_{\theta}$ in the tangential injection reservoir.

$p_{1}^{\prime}=\frac{\mathrm{d} p_{1}^{\prime}}{\mathrm{d} t}\left(t-t_{\text {open }}\right) \simeq \frac{c_{1}^{2} \dot{m}_{\theta}}{V_{\text {set }}}\left(t-t_{\text {open }}\right)$

were $t_{\text {open }}=2.5 \mathrm{~ms}$ is the moment the tangential-injection valve is opened. This model is compared to measurements of $p_{1}^{\prime}$ in Fig. 5a.

Due to the adiabatic increase in upstream reservoir pressure, $p_{1}^{\prime}$, there will be an increase in upstream reservoir density $\rho_{1}$ :

$\frac{\rho_{1}^{\prime}}{\rho_{1}}=\frac{p_{1}^{\prime}}{\gamma p_{1}}$.

For the upstream reservoir speed of sound, one has

$\frac{c_{1}^{\prime}}{c_{1}}=\frac{T_{1}^{\prime}}{2 T_{1}}=\left(\frac{\gamma-1}{2 \gamma}\right) \frac{p_{1}^{\prime}}{p_{1}}$.

Applying a quasi-steady approximation, yields

$\frac{\dot{m}_{1}^{\prime}}{\dot{m}_{1}}=\frac{\dot{m}_{2}^{\prime}}{\dot{m}_{2}}=\frac{u_{2}^{\prime}}{u_{2}}+\frac{\rho_{2}^{\prime}}{\rho_{2}}=\frac{\rho_{1}^{\prime}}{\rho_{1}}+\frac{c_{1}^{\prime}}{c_{1}}=\left(\frac{\gamma+1}{2 \gamma}\right) \frac{p_{1}^{\prime}}{p_{1}}$

The fluctuation in $\dot{m}_{2}^{\prime}$ is related to the downstream velocity and density fluctuations, as follows

$\frac{\dot{m}_{2}^{\prime}}{\dot{m}_{2}}=\frac{u_{2}^{\prime}}{u_{2}}+\frac{\rho_{2}^{\prime}}{\rho_{2}}$

Downstream of the nozzle one assumes anechoic conditions. Thus, the downstream pressure fluctuation $p_{2}^{\prime}$ is given by

$p_{2}^{\prime}=\rho_{2} c_{2} u_{2}^{\prime}$.

Using $\rho_{2} c_{2}^{2}=\gamma p_{2}$, one obtains

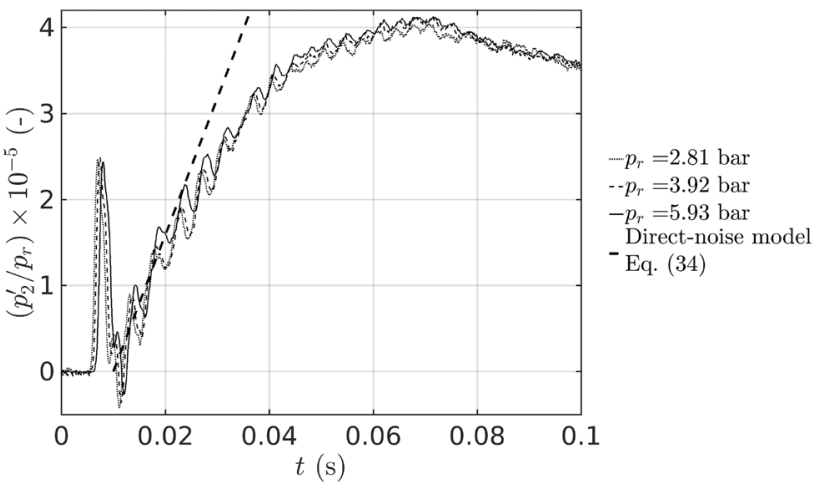

Fig. $10 p_{2}^{\prime} / p_{r}$, where $p_{r}$ is the radial injection reservoir pressure, as a function of time. The measurements were performed with the radial injection port $185 \mathrm{~mm}$ upstream from the nozzle inlet. A valve trigger time of $100 \mathrm{~ms}$ was used. The experimental data are compared to the direct-noise model [Eq. (34); thick-dashed line]

$\frac{\rho_{2}^{\prime}}{\rho_{2}}=\frac{u_{2}^{\prime}}{c_{2}}$.

Upon substitution in Eq. (30), one finds

$\frac{\dot{m}_{2}^{\prime}}{\dot{m}_{2}}=\frac{u_{2}^{\prime}}{u_{2}}\left(1+\frac{u_{2}}{c_{2}}\right) \simeq \frac{u_{2}^{\prime}}{u_{2}}$

where $u_{2} / c_{2}<<1$ was used. Combining this result with equations 26 and 29, yields

$p_{2}^{\prime}(t)=\rho_{2} c_{2} u_{2}^{\prime}(t)=\frac{p_{2}}{p_{1}} c_{2} u_{2}\left(\frac{\gamma+1}{2}\right)\left(\frac{\dot{m}_{\theta}}{V_{\text {set }}}\right)\left(t-t_{0}\right)$

where the ideal gas relationship $\rho_{1} c_{1}^{2}=\gamma p_{1}$, and the low Mach number approximation $T_{1}=T_{2}$ were used. $t_{0}$ corresponds to the arrival time at the microphone of the initial direct sound pulse (caused by the opening of the injection valve).

In Fig. 10, the $p_{2}^{\prime}$ signal due to radial injection of air is compared to that predicted by Eq. (34). The model predicts the measurements quite accurately for $t<30 \mathrm{~ms}\left(t_{0}=10 \mathrm{~ms}\right)$.

As the valve closes, the reservoir pressure perturbation $p_{1}^{\prime}$ has reached a maximum value, which is still small compared to the reservoir pressure $p_{1}=1.12$ bar. The decay of $p_{1}^{\prime}$ is in linear approximation described by the mass balance:

$\frac{V_{\text {set }}}{c_{1}^{2}} \frac{\mathrm{d} p_{1}^{\prime}}{\mathrm{d} t}=-\dot{m_{1}} \frac{\gamma+1}{2 \gamma} \frac{p_{1}^{\prime}}{p_{1}}=-\frac{\gamma+1}{2} \frac{u_{1} A_{1}}{c_{1}^{2}} p_{1}^{\prime}$

Thus, the reservoir pressure perturbation decays exponentially with time after the valve is closed. This decay has a time constant $2 V_{\text {set }} /\left((\gamma+1) u_{1} A_{1}\right) \simeq 1.0 \mathrm{~s}$. The delay of $3 \mathrm{~s}$ between each experiment allows the reservoir pressure perturbation to decrease to $5 \%$ of its maximum value. 
Acknowledgements The authors thank the DLR's technical staff members Nico Seiffert and Lech Modrzejewski for their support. Lionel Hirschberg thanks Catherine Lemaitre and Assa Ashuach for their support.

Author contributions All authors have materially participated in the research and/or article preparation. Hirschberg is the principal investigator for the reported work. He is the lead and corresponding author. Hirschberg performed the reported experiments and analysis thereof. Bake and Knobloch assisted Hirschberg with measurements. Bake, Rudolphi, Kruck, and Klose were involved in the design and manufacture of the experimental setup used to produce the reported data. Hulshoff provided essential support in the analysis of the data, as well as in the redaction of the text.

Funding Open Access funding enabled and organized by Projekt DEAL.

\section{Declarations}

Conflict of interest The authors declare that they have no conflicts of interest.

Open Access This article is licensed under a Creative Commons Attribution 4.0 International License, which permits use, sharing, adaptation, distribution and reproduction in any medium or format, as long as you give appropriate credit to the original author(s) and the source, provide a link to the Creative Commons licence, and indicate if changes were made. The images or other third party material in this article are included in the article's Creative Commons licence, unless indicated otherwise in a credit line to the material. If material is not included in the article's Creative Commons licence and your intended use is not permitted by statutory regulation or exceeds the permitted use, you will need to obtain permission directly from the copyright holder. To view a copy of this licence, visit http://creativecommons.org/licenses/by/4.0/.

\section{References}

Anthoine J, Buchlin JM, Hirschberg A (2002) Effect of nozzle cavity on resonance in large SRM: theoretical modeling. J Propuls Power 18(2):304-311. https://doi.org/10.2514/2.5935

Carpenter PW (1976) A general one-dimensional theory of compressible inviscid swirling flows in nozzles. Aeronaut Q 27:201-216

Dotson KW, Koshigoe S, Pace KK (1997) Vortex shedding in a large solid rocket motor without inhibitors at the segmented interfaces. J Propuls Power 13(2):197-206. https://doi.org/10.2514/2.5170

Dowling AP, Mahmoudi Y (2015) Combustion noise. Proc Combust Inst 35(1):65-100. https://doi.org/10.1016/j.proci.2014.08.016

Dutton JC (1989) Correlation of nozzle performance degradation due to swirl. J Propuls Power 5(1):126-128. https://doi.org/10.2514/3. 23125

Ffowcs Williams JE, Howe MS (1975) The generation of sound by density inhomogeneities in low mach number nozzle flows. J Fluid Mech 70(3):605-622. https://doi.org/10.1017/S00221120750022 24
Gany A, Mor M, Goldman C (2005) Analysis and characteristics of choked swirling nozzle flows. AIAA J 43(10):2177-2181. https:// doi.org/10.2514/1.16887

Hirschberg L, Hulshoff SJ (2020) Lumped-element model for vortexnozzle interaction in solid rocket motors. AIAA J 58(7):32413244. https://doi.org/10.2514/1.J058673

Hirschberg L, Hulshoff SJ, Collinet J, Schram C, Schuller T (2018a) Vortex nozzle interaction in solid rocket motors: a scaling law for upstream acoustic response. J Acoust Soc Am 144(1):EL46EL51. https://doi.org/10.1121/1.5046441

Hirschberg L, Schuller T, Collinet J, Schram C, Hirschberg A (2018b) Analytical model for the prediction of pulsations in a cold-gas scale-model of a solid rocket motor. J Sound Vib 19:445-368. https://doi.org/10.1016/j.jsv.2018.01.025

Hirschberg L, Hulshoff SJ, Collinet J, Schram C, Schuller T (2019) Influence of nozzle cavity on indirect vortex- and entropy-sound production. AIAA J 57(7):3100-3103. https://doi.org/10.2514/1. J058138

Hirschberg L, Bake F, Knobloch K, Hulshoff SJ (2021a) Swirl-nozzle interaction experiments: influence of injection-reservoir pressure and injection time. AIAA J 59(7):2806-2810. https://doi.org/10. 2514/1.J060291

Hirschberg L, Hulshoff SJ, Bake F (2021b) Sound production due to swirl-nozzle interaction: model-based analysis of experiments. AIAA J 59(4):1269-1276. https://doi.org/10.2514/1.J059669

Hulshoff SJ, Hirschberg A, Hofmans GCJ (2001) Sound production of vortex nozzle interactions. J Fluid Mech 439:335-352. https://doi. org/10.1017/S0022112001004554

Ihme M (2017) Combustion and engine-core noise. Annu Rev Fluid Mech 49:277-310. https://doi.org/10.1146/annur ev-fluid-122414-034542

Kings N (2015) Indirect combustion noise: Experimental investigation of the vortex sound generation in nozzle flows. Ph.D. thesis, Technische Universität Berlin

Kings N, Bake F (2010) Indirect combustion noise: noise generation by accelerated vorticity in a nozzle flow. Int J Spray Combust Dyn 2(3):253-266. https://doi.org/10.1260/1756-8277.2.3.253

Marble FE, Candel SM (1977) coustic disturbance from gas non-uniformities convected through a nozzle. J Sound Vib 55:225-243. https://doi.org/10.1016/0022-460X(77)90596-X

Morgans AS, Duran I (2016) Entropy noise: a review of theory, progress and challenges. Int J Spray Combust Dyn 8(4):285-298. https://doi.org/10.1177/1756827716651791

Neuhaus D, Röhle I (2006) Schnellschaltende ventile für anwendungen in der luft und raumfahrt. In: Deutscher Luft- und Raumfahrtkongress

Strahle WC (1971) On combustion generated noise. J Fluid Mech 49(2):399-414. https://doi.org/10.1017/S0022112071002167

van Holten T, Heiligers M, Jaeken A (2009) Choking phenomena in a vortex flow passing a laval tube: an analytical treatment. J Fluids Eng 131(4):041201. https://doi.org/10.1115/1.3089532

Publisher's Note Springer Nature remains neutral with regard to jurisdictional claims in published maps and institutional affiliations. 


\section{Authors and Affiliations}

Lionel Hirschberg $^{1}$ (1) $\cdot$ Friedrich Bake ${ }^{1} \cdot$ Karsten Knobloch $^{1} \cdot$ Angelo Rudolphi $^{1} \cdot$ Sebastian Kruck ${ }^{1}$. Oliver Klose ${ }^{1}$. Steven J. Hulshoff ${ }^{2}$

Lionel Hirschberg

1.hirschberg@me.com

1 Deutsches Zentrum für Luft- und Raumfahrt (DLR;

German Aerospace Center), Institute of Propulsion

Technology, Engine Acoustics, Mueller-Breslau-Straße 8,

Charlottenburg, Berlin 10623, Germany
2 Faculty of Aerospace Engineering, Delft University of Technology, Kluyverweg 1, 2629HS Delft, The Netherlands 